\title{
Comparing the Efficacy of 8 Weeks Treatment of Cipram® and its Generic Citalopram in Patients With Mixed Anxiety-Depressive Disorder
}

\author{
Hasan Khoonsari ${ }^{1}$; Mohammad Bagher Oghazian ${ }^{1}$; Mona $\operatorname{Kargar}^{2}$; Mahdiyeh Moin ${ }^{3}$; \\ Hossein Khalili ${ }^{1}$; Abbas Alimadadi ${ }^{4}$; Hassan Torkamandi ${ }^{5}$; Padideh Ghaeli ${ }^{6, *}$ \\ ${ }^{1}$ Department of Clinical Pharmacy, Faculty of Pharmacy, Tehran University of Medical Sciences, Tehran, IR Iran \\ ${ }_{2}^{2}$ Research Center for Rational Use of Drugs, Faculty of Pharmacy, Tehran University of Medical Sciences, Tehran, IR Iran \\ ${ }_{4}^{3}$ Department of Psychiatry, Roozbeh Hospital, School of Medicine, Tehran University of Medical Sciences, Tehran, IR Iran \\ ${ }_{5}^{4}$ University of Applied Science and Technology, Tehran, IR Iran \\ 5 Pharmaceutical Care Department, Shariati Hospital, Tehran University of Medical Sciences, Tehran, IR Iran \\ 6 Pharmaceutical Care Department, Shariati Hospital, Tehran University of Medical Sciences, Tehran, IR Iran
${ }_{\text {Psychiatry and Psychology Research Center, Faculty of Pharmacy, Roozbeh Hospital, Tehran University of Medical Sciences, Tehran, IR Iran }}$ \\ *Corresponding author: Padideh Ghaeli, Psychiatry and Psychology Research Center, Faculty of Pharmacy, Roozbeh Hospital, Tehran University of Medical Sciences, P. O. BOX: \\ 1333715914 , Tehran, IR Iran. Tel: +98-2155412222, Fax: +98-2155419113, E-mail: mmppg@yahoo.com
}

Received: February 1, 2014; Revised: November 6, 2014; Accepted: November 28, 2014

\begin{abstract}
Background: Patients with mixed anxiety-depressive disorder (MADD) suffer both anxiety and depression. Antidepressants, especially, selective serotonin reuptake inhibitors are among agents of choice for treating this condition.

Objectives: This study compared the efficacy of Cipram® with its generic, citalopram.

Patients and Methods: Forty adult outpatients (between 18 to 55 years of age) with a diagnosis of MADD who met the trial criteria, entered this double-blind, randomized study. Subjects were assigned to receive either generic citalopram or Cipram® for 8 weeks. Hamilton Rating Scale for Depression (HAM-D) and Hamilton Rating Scale for Anxiety (HAM-A) were utilized to assess depression and anxiety at baseline, weeks 4 and 8 of the study. Statistical analysis was performed using SPSS 14.0.

Results: Twenty patients received citalopram (mean dosages of $22 \mathrm{mg} /$ day during the first 4 weeks and $33 \mathrm{mg} /$ day during weeks 4 to 8 ) and 20 received Cipram $®$ (mean dosages of $22 \mathrm{mg} /$ day during the first 4 weeks and $29 \mathrm{mg} /$ day during weeks 4 to 8 ). Both treatments were noted to be effective in improving the symptoms of MADD at weeks 4 and 8 . The mean differences of HAM-D and HAM-A between Citalopram and Cipram® groups were significantly different at the end of week 4 (HAM-D: P=0.038, HAM-A: P=0.025), but not at the end of week 8 (HAM-D: $\mathrm{P}=0.239$, HAM-A: $\mathrm{P}=0.204$ ). Both medications were tolerated well by the patients.

Conclusions: This study suggests that the efficacy of citalopram is similar to that of Cipram $®$ in the treatment of MADD after 8 weeks. Meanwhile, Cipram® may reduce depression and anxiety quicker than its generic, citalopram.
\end{abstract}

Keywords: Citalopram; Anxiety; Depressive Disorder; Serotonin Uptake Inhibitors

\section{Background}

Mixed anxiety depression was initially introduced by Overall and colleagues in 1966 and was later reported by Paykel in 1971 (1). Patients with Mixed anxiety-depressive disorder (MADD) have a combination of both anxiety and depressive symptoms (2). According to ICD-10, MADD is considered when the patient suffers from symptoms of both anxiety and depression, but neither is predominantly significant, nor meets the diagnostic criteria as a separate disorder (3). Most trials in the literature have studied the effects of medications on significant depression or anxiety even though many patients do not full fill the criteria for these two psychiatric disorders. Therefore studies on the effects of medications in patients with MADD seem to be of importance.

In a study by Kara et al. (1), it was noted that patients diagnosed with MADD had less depressive and more anxiety symptoms when compared with those suffering major depressive disorder (MDD). This study did not show any differences in the results of both dexamethasone suppression and thyroid function tests between the two groups. However, in MADD patients, proportional overactivation of hypothalamic-pituitary-adrenal (HPA) axes was noted after stabilization of severe symptoms. The prevalence of MADD is a subject of conflicts. The prevalence of MADD in a study including 21,644 primary care patients in Italy was about 1.8\% (2). Based on the data in the Netherlands Mental Health and Incidence Study, Spijker et al. (4) reported a 12-month prevalence of MADD of about $0.6 \%$ in the general population. It has been proposed that stressful life events are not the predisposing factors for MADD (1). The mainstay of pharmacotherapy in this disorder consists of combination of antidepressants and anxiolytics (5). Because of their better tolerability (6), similarity in terms of efficacy and better safety in overdose, second-generation antidepressants including Selective Serotonin Reuptake Inhibitors (SSRIs) have

Copyright (C) 2015, Mazandaran University of Medical Sciences. This is an open-access article distributed under the terms of the Creative Commons Attribution-NonCommercial 4.0 International License (http://creativecommons.org/licenses/by-nc/4.0/) which permits copy and redistribute the material just in noncommercial usages, provided the original work is properly cited. 
gradually substituted tricyclic antidepressants (TCAs) since mid-1980s and became the first-line medications for the treatment of depression (7). In fact the introduction of SSRIs was the beginning of a great therapeutic era in psychopharmacology (8). Within a relatively short period of time, different companies developed drugs of this family. The first of which was fluoxetine manufactured by Eli Lilly pharmaceuticals. Afterwards, sertraline, paroxetine, fluvoxamine, citalopram and lately escitalopram were developed. SSRIs became the most dominant prescribed antidepressant medications in US market in 2005 and 60\% of the Medicaid covered prescription of antidepressants contained them. However, use of SSRIs in the treatment of MADD has not been evaluated widely. Since a considerable amount of total medical expenditure is made up of pharmaceutical expenditure, recently changing from more expensive brands to their generic drugs have been widely encouraged by authorities (9-11); this is considered a way to reduce healthcare expenditure (11). For example in the United States of America, total budget devoted to antidepressants increased in a 13 year period (1991 - 2004) from $159 \$$ million to $2.26 \$$ billion but after the entrance of cheaper generic fluoxetine and paroxetine in 2001 and 2003 , respectively, this amount decreased to $1.99 \$$ billion in 2005 (6). Based on an international review of the literature, Simoens performed a descriptive policy analysis regarding substitution of brand name drugs with their generics and reported that switching to generic drugs may decrease public expenditure on brand name drugs by $21 \%$ (12).

Saving money by using generic drugs can be dedicated to the treatment of more patients and can provide resources for other treatment modalities (11). This study aimed to compare the efficacy of branded citalopram, Cipram $\circledast$ manufactured by Lundbeck pharmaceutical company with generic citalopram made by Sobhan Darou for the treatment of MADD. Lundbeck pharmaceutical company has been known as the first manufacturer of citalopram in the world. The patent of this drug has expired as of 2003 and as a result, this drug could be manufactured by other pharmaceutical companies under the related regulatory law (13). According to the world health organization (WHO), "a generic product is a drug that is manufactured by an authorized manufacturer under nonproprietary or approved names" (14). WHO also mentions that "a generic product may be marketed under its generic name or brand name" (14). Citalopram made by Sobhan Darou (15) as a generic drug was selected for comparison with Cipram $®$ in this study due to the reasonable number of prescriptions filled for this medication in Iran.

\section{Objectives}

The present study was designed to compare the efficacy of Cipram $\AA$ made by Lundbeck with its generic citalopram made by Sobhan Darou in the treatment of MADD.

\section{Materials and Methods}

This 8-week, double-blind, randomized trial (ID: IRCT201405127202N8 that was registered in www.irct. ir). Adult patients who suffered MADD based on the "text revision" of Diagnostic and Statistical Manual of Mental Disorders, Fourth edition (DSM-IV-TR) (16) were screened at the outpatient clinic of Roozbeh Hospital affiliated with Tehran University of Medical Sciences from April 2006 through September 2007. As shown below, the sample size of 40 patients (20 in each group) was calculated to detect at least a change of 7 in mean HAM-A or HAM-D between the two groups at $\alpha$ (2-sided) $=0.05$ and with $\mathrm{a} \%$ power of 80 (Equation 1$)$, where $\mathrm{d}=$ M1 - M2 = 7:

(1) $2\left(Z_{\left(1-\frac{\alpha}{2}\right)}+Z_{(1-\beta)}\right)^{2} \frac{S^{2}}{d^{2}}=2(1.96+0.84)^{2} \times \frac{8^{2}}{7^{2}}=19.59$

Patients were between 18 and 55 years old and had Hamilton Rating Scale for Depression (HAM-D) and Hamilton Rating Scale for Anxiety (HAM-A) scores of less than 24.

The exclusion criteria included history of other psychiatric disorders including personality disorders, mental retardation, organic brain syndrome, neurological disorders, unstable medical situations including cardiovascular, hepatic, renal, endocrine or hematological disorders, alcohol or drug dependence, having suicidal idea, pregnancy and lactation. Patients who received antidepressants within 3 weeks prior to the initiation of the study as well as those who underwent ECT within six months before the start of the trial were also excluded. Besides, patients on other drugs that could affect depression or anxiety were excluded from the study. The study was approved by ethics committee of Tehran University of Medical Sciences. All patients provided written informed consent prior to study initiation. At their entry in the study, patients were randomized by permuted-blocked method to receive either citalopram or Cipram®. The medications were packed and labeled as group 1 or 2 in identical small paper bags by a pharmacy technician who was not aware of the study details. Study drugs were started at $10 \mathrm{mg}$ per day for the first 3 days. Then, the daily dose was increased to $20 \mathrm{mg}$ or higher based on the patients' responses and tolerability. Maximum dose allowed in the trial was $40 \mathrm{mg}$ per day.

Patients were assessed by using standardized, 21 item HAM-D and HAM-A at baseline, 4 and 8 weeks after starting medication. The mean differences in HAM-D and HAM-A scores from baselines were the basis of the main outcome measures of response to treatment. Statistical analyzes were performed using SPSS 14.0 (Inc., Chicago, Illinois, USA). Paired Student's t-test and one-way repeated measures ANOVA test were used as statistical tools to evaluate the differences between groups and differences in each group at baseline, 4 and 8 weeks after beginning of the study. P values less than 0.05 were considered significant. 


\section{Results}

Twenty patients received citalopram and 20 received Cipram $®$ in this study (Figure 1). Patients in the citalopram group received a mean dosage of $22 \mathrm{mg} /$ day during the first 4 weeks and a mean dosage of $33 \mathrm{mg} /$ day throughout weeks 4 to 8 . In the Cipram® group, patients received a mean dosage of $22 \mathrm{mg}$ /day during the first 4 weeks and a mean dosage of $29 \mathrm{mg} /$ day throughout weeks 4 to 8 . Table 1 summarizes the demographic data of patients in both groups. Age and gender of the participants were not found to be significantly different. Furthermore, no significant difference was noted between the two groups for baseline HAM- D (18.35 \pm 2.03 vs. $18.05 \pm 2.06 ; \mathrm{P}=0.888)$ and HAM-A (23.20 \pm 3.92 vs. $23.55 \pm 6.03 ; \mathrm{P}=0.933)$ scores between Citalopram and Cipram® groups, respectively. Using Paired Student's ttest mean differences of HAM-D scores between baseline and weeks 4 and baseline and week 8 in the citalopram group were $4.40(\mathrm{P}<0.001)$ and $7.95(\mathrm{P}<0.001)$, respectively and mean difference between weeks 4 to 8 was 3.55 ( $\mathrm{P}<0.001)$. In Cipram $®$ group the mean differences of HAM-D scores between baseline and weeks 4 and 8 were $6.50(\mathrm{P}<0.001)$ and $9.95(\mathrm{P}<0.001)$, respectively and mean difference between weeks 4 to 8 was 3.45 ( $\mathrm{P}<$ 0.001). In terms of differences of HAM-A in citalopram group these data obtained: mean differences of HAM-
A scores between baseline and weeks 4, between baseline and weeks 8 and between weeks 4 to 8 was 5.05 (P $<0.001)$, $9.00(\mathrm{P}<0.001)$ and $4.00(\mathrm{P}<0.001)$, respectively. Mean differences of HAM-A in Cipram $₫$ group at the same intervals were $8.35(\mathrm{P}<0.001)$ and $12.40(\mathrm{P}<$ $0.001)$ and $4.05(\mathrm{P}=0.002)$, respectively. Therefore, these results showed a significant effect of both treatments on the HAM-D and HAM-A scales at the end of the fourth and eighth weeks of the study which supports the effectiveness of both treatment modalities after 8 week. Oneway repeated measures ANOVA on difference between mean differences in the HAM-D and HAM-A scales between 4 th week of treatment and baseline showed significant differences between the two treatment groups (Table 2). This significant difference was in favor of Cipram® in terms of reducing both depression and anxiety symptoms during the first month of therapy. Interestingly the mean differences in HAM-D and HAM-A scores between groups were not significantly different at the end of week 8 which were assessed with the same test. Thus, in both groups patients showed similar improvement in mean HAM-D and HAM-A scores at the end of 8 week in spite of the significant difference at the end of 4 th week. The trend in reduction of HAM-A and HAM-D in both groups are shown in figure 2 and 3 respectively.

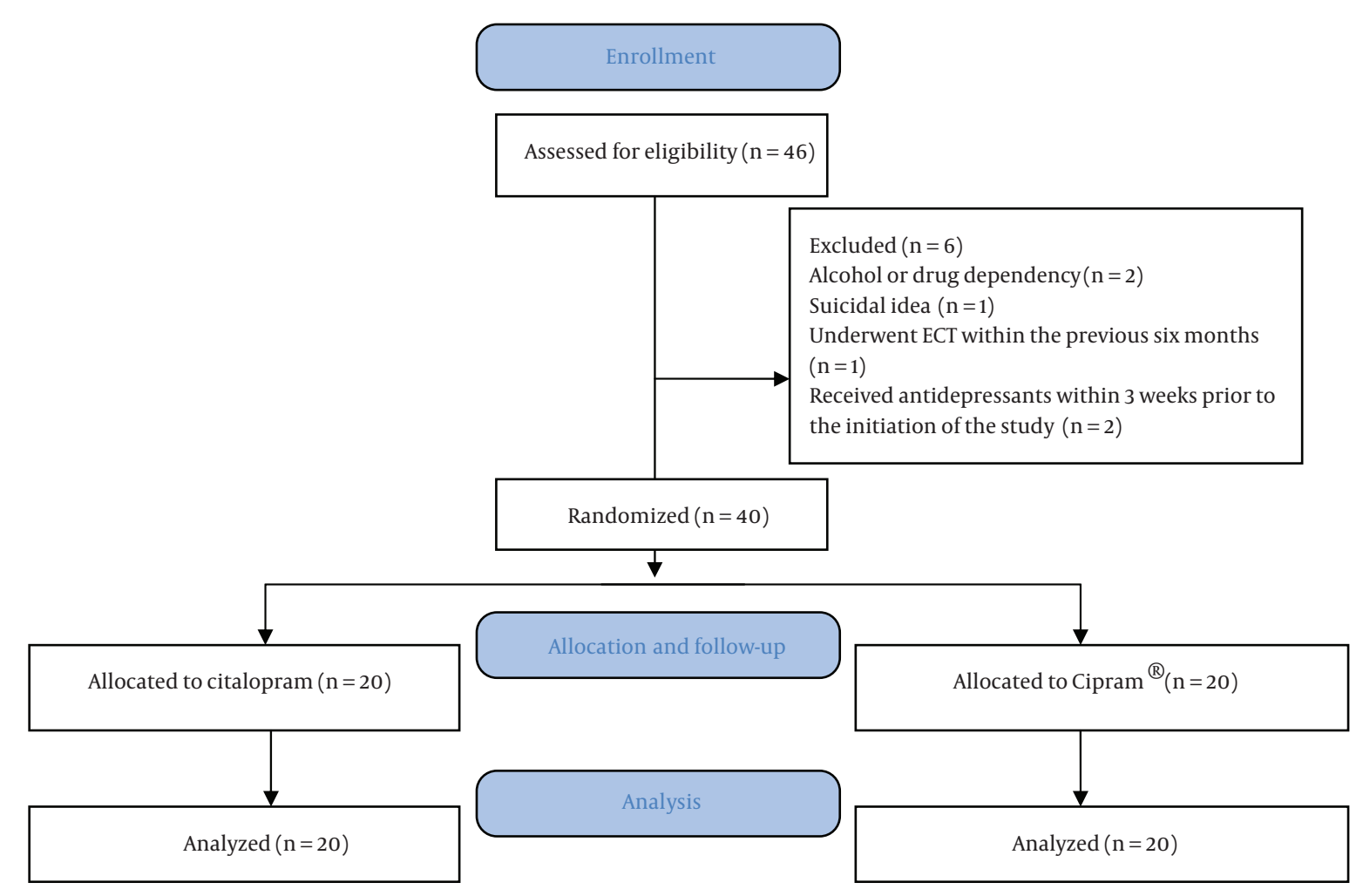

Figure 1. Consort Flow Diagram of Patient Recruitment and Study Completion 
Khoonsari H et al.

\begin{tabular}{|c|c|c|c|}
\hline Variables & Group $1^{b}$ & Group $2^{C}$ & PValue \\
\hline Age, y & & & 0.613 \\
\hline $\operatorname{Mean} \pm S D$ & $33.10 \pm 6.21$ & $36.10 \pm 5.66$ & \\
\hline Range & $19-55$ & $21-55$ & \\
\hline \multicolumn{4}{|l|}{ Gender } \\
\hline Female, n & 18 & 17 & $1.00^{d}$ \\
\hline
\end{tabular}

Table 2. Difference Between Mean Differences of HAM-A and HAM-D Scores in Groups 1 and 2 in Different time Schedule Between week 4 and Baseline, week 8 and Baseline and Weeks 8 and 4

\begin{tabular}{cc}
\hline Scale & $\begin{array}{c}\text { Mean Differences } \text { PValue }^{\mathrm{C}} \\
\text { of Group 1 }\end{array}$ \\
Group 2 $^{\text {b }}$ \\
\hline
\end{tabular}

\section{HAM-A}

$\begin{array}{lll}\text { Between Week } 4 \text { and Baseline } & 3.30 & 0.025^{\mathrm{d}} \\ \text { Between Week } 8 \text { and baseline } & 3.40 & 0.204 \\ \text { Between Week } 8 \text { and Week } 4 & 1.00 & 0.997\end{array}$

HAM-D

\begin{tabular}{|lcc|}
\hline Between Week 4 and Baseline & 2.10 & $0.038^{\mathrm{d}}$ \\
\hline Between Week 8 and baseline & 2.00 & 0.239 \\
\hline Between Week 8 and Week 4 & 0.10 & 0.995 \\
\hline
\end{tabular}

a Group 1= Patients on citalopram manufactured by Sobhan Darou.

b Group 2= Patients on Cipram @ manufactured by Lundbeck.

${ }^{\text {c }}$ P value $<0.05$ is considered significant.

$\mathrm{d}$ Shows significant difference between groups 1 and 2 .

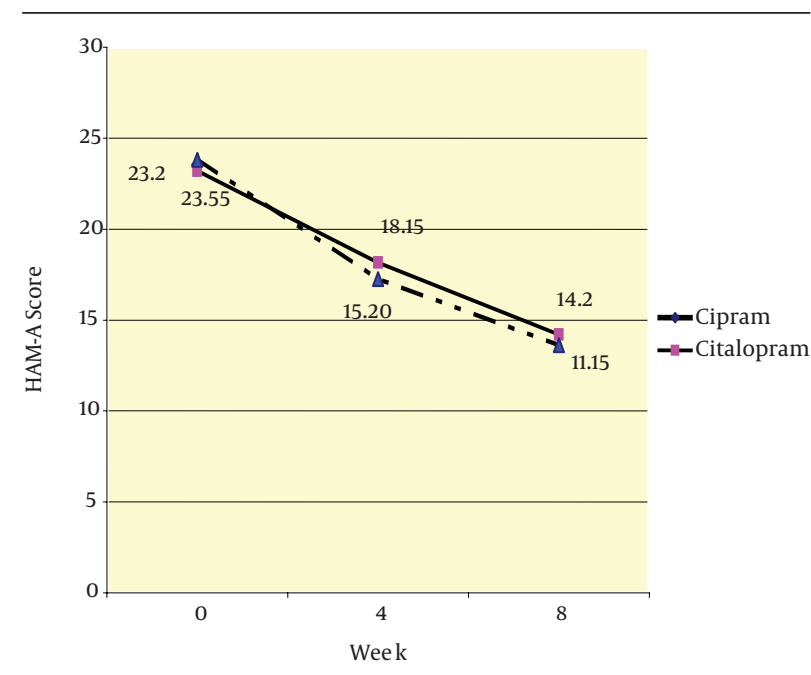

Figure 2. The Trend in Reduction of HAM-A Patients Receiving Citalopram and Cipram® During the Study

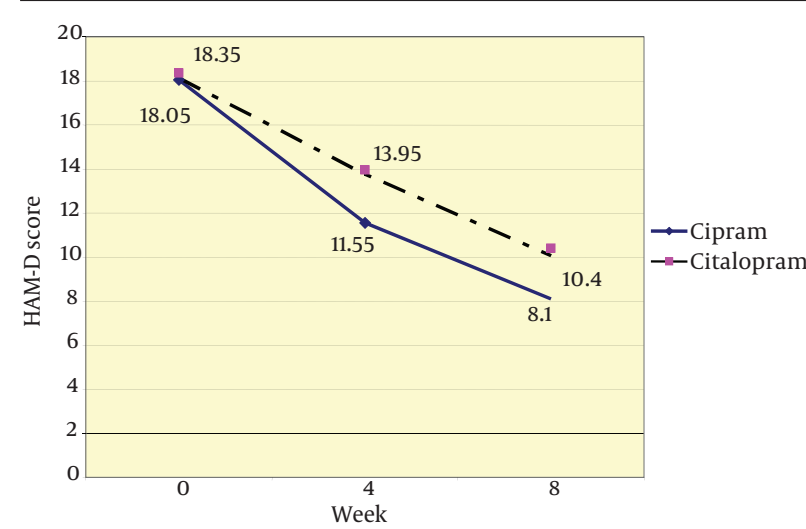

Figure 3. The Trend in Reduction of HAM-D Patients Receiving Citalopram and Cipram® During the Study

\section{Discussion}

Citalopram is considered to have the most selective serotonin reuptake inhibition among SSRIs $(8,17)$. SSRIs originally were developed for the treatment of depression, depression; however, with increased knowledge about the serotonin role in psychiatric disorders has led to the broader applications of these drugs in different disorders (6). To the authors' knowledge, the efficacy of SSRIs in the treatment of MADD has not been evaluated widely in the treatment of MADD. As mentioned above, both treatment modalities in our study significantly reduced the HAM-A and HAM-D at 8 week which shows citalopram efficacy in this disorder. The outcome of our study is in accordance with the study of Carrasco et al. (5) who have evaluated sertraline in the treatment of MADD. In that 8-week study, 29 out of 36 patients (76\%) were responders to sertraline. Prescribing a generic drug instead of its brand-name may be potentially cost-saving (6), however, concerns regarding several remarkable points may affect patients' and health care providers' trust in generic drugs negatively (18). Theoretically, a generic drug is a pharmaceutical product that is launched with no intellectual property or other protection after expiry date of the patent or other exclusive rights of the innovator product (19). As a general rule the generic drug must contain the same active ingredient and be available with the same pharmaceutical dosage form and dose. Also demonstration of the bioequivalence of the generic drug within an acceptable range with the original brand is required (20). Despite the lack of evidence for inferiority of generic drugs to their branded counterparts, many patients may resist changing from a brand name drug to its generic equivalent (10). Even some physicians may not believe in the same efficacy of the brand name and generic drugs. For example, physicians participated in a survey in Finland, doubted the equivalency of certain generic medications in terms of efficacy and safety (11). Several other studies have expressed the existence of a con- 
troversy about equivalency of generic and brand drugs in terms of effects and safety in different illnesses (21-24).

In a case study of six patients with depression, Yu et al. (25) reported that Prozac® was more effective and resulted in fewer side effects when compared to its generic fluoxetine. In our study, in spite of significant differences of both HAM-D and HAM-A between the groups at the end of week 4 in favor of Cipram®, no significant differences were noted at the end of week 8 . This can be interpreted as a more rapid onset of effects with Cipram®. However, the same efficacy is obtained with citalopram when the duration of treatment is long enough. Since the cost benefit ratio is an important issue in selecting a medication for a patient, especially, someone who suffers from a chronic disorder, our study suggests that generic citalopram may be preferred over Cipram $₫$ to be initiated in most patients. Moreover, it should be noted that patients receiving Cipram® were on the lower doses of the drug when compared with those who received generic citalopram. This may suggest that Cipram $®$ in lower doses is as efficacious as higher doses of its generic. One important limitation in the present study was the fact that a small number of patients with a diagnosis of MADD were compared in this trial.

This study suggests that the efficacy of Cipram® and its generic drug seems to be similar in the treatment of MADD after 8 weeks. However, Cipram $®$ may reduce depression and anxiety quicker than its generic, citalopram. Studies with a larger number of patients and for a longer duration may be needed to confirm the results of this study.

\section{Acknowledgements}

This research was a part of Dr Hasan khoonsari's pharmacy thesis project and was supported by Psychiatry and Psychology Research Center affiliated by Tehran University of Medical Sciences and Health Services (Grant number 85-01-44-3566). This trial was registered at the Iranian Clinical Trial site identified by the following code: IRCT201405127202N8). The authors would like to thank Shahin Akhondzadeh (Ph.D), Firoozeh Raisi (MD), Vandad Sharifi (MD), Arghavan Sadeghi (MD), Vahid Iravani (MD), H. Jalaeyan (PharmD) and Mr. Abbas Kamalipour (R.N). We would also like to thank the staff at Roozbeh hospital pharmacy, nursing and laboratory.

\section{Authors' Contributions}

Hasan Khoonsari participated in designing the trial, collected and evaluated the clinical data; he also participated in drafting the manuscript. Mohammad Bagher Oghazian and Mona Kargar were involved in the original designing of the proposal, and also in drafting and revising the manuscript. Mahdiyeh Moin was involved in designing the study, and was responsible for recruiting patients. Hossein Khalili interpreted the clinical data and performed the statistical analysis. Abbas Alimadadi was involved in evaluating all patients and collecting the clinical data. Hassan Torkamandi reassessed the clinical data, reviewed the statistical analysis and interpreted them. Padideh Ghaeli supervised the trial and was involved in designing the study as well as coordinating the project, drafting and revising the manuscript. All authors read and approved the final manuscript.

\section{Clinical Trial Registration Code}

\section{IRCT201405127202N8.t}

\section{Funding/Support}

This research was a part of Dr. Hasan Kkhoonsari's pharmacy thesis project and was supported by Psychiatry and Psychology Research Center affiliated by Tehran University of Medical Sciences and Health Services (Grant number 85-01-44-3566).

\section{Declaration of Interest}

None declared.

\section{References}

1. Mixed anxiety-depressive disorder and major depressive disorder: comparison of the severity of illness and biological variables. Paykel. Kara S, Yazici KM, Gulec C, Unsal I. Psychiatry Res. 1971;94(1):59.

2. Assessing mixed anxiety-depressive disorder. A national primary care survey. Balestrieri M, Isola M, Quartaroli M, Roncolato M, Bellantuono C. Psychiatry Res. 2010;176(2-3):197.

3. World Health Organization . International Statistical Classification of Diseases and Related Health Problems 10th Revision. WHO; 2010. Available from: http://apps.who.int/classifications/ apps/icd/icd10online

4. Who is MADD? Mixed anxiety depressive disorder in the general population. Spijker J, Batelaan N, de Graaf R, Cuijpers P. J Affect Disord. 2010;121(1-2):180.

5. Sertraline in the treatment of mixed anxiety and depression disorder. Carrasco JL, Diaz-Marsa M, Saiz-Ruiz J. J Affect Disord. 2000;59(1):67.

6. Utilization, price, and spending trends for antidepressants in the US Medicaid Program. Chen Y, Kelton CM, Jing Y, Guo JJ, Li X, Patel NC. Res Social Adm Pharm. 2008;4(3):244.

7. Comparative benefits and harms of second-generation antidepressants: background paper for the American College of Physicians. Gartlehner G, Gaynes BN, Hansen RA, Thieda P, DeVeaughGeiss A, Krebs EE, et al. Ann Intern Med. 2008;149(10):734.

8. Role of selective serotonin reuptake inhibitors in psychiatric disorders: a comprehensive review. Vaswani M, Linda FK, Ramesh S. Prog Neuropsychopharmacol Biol Psychiatry. 2003;27(1):85.

9. Acid-suppressive effects of generic omeprazole: comparison of three brands of generic omeprazole with original omeprazole. Shimatani T, Inoue M, Kuroiwa T, Xu J, Mieno H, Tazuma S. Dig Liver Dis. 2006;38(8):554.

10. A prospective multicenter study of the effect of patient education on acceptability of generic prescribing in general practice. Valles JA, Barreiro M, Cereza G, Ferro JJ, Martinez MJ, Escriba JM, et al. Health Policy. 2003;65(3):269.

11. A survey exploring knowledge and perceptions of general practitioners towards the use of generic medicines in the northern state of Malaysia. Chua GN, Hassali MA, Shafie AA, Awaisu A. Health Policy. 2010;95(2-3):229.

12. Developing competitive and sustainable Polish generic medicines market. Simoens S. Croat Med J. 2009;50(5):440. 


\section{Khoonsari H et al.}

13. [cited October 22];Wikipedia Citalopram. Available from: http:// en.wikipedia.org/wiki/Citalopram.

14. WHO Regional Office for Africa . Management of Drugs at Health Centre Level - Training Manual. Brazzaville: WHO;2004. Available from: http://apps.who.int/medicinedocs/en/d/Js7919e/11.html.

15. Citalopram. Sobhan Darou; [cited October 22]. 2012 . Available from: http://www.sobhandarou.com/CNS.html?M=PRODUCTS\& MC $=$ SHOWPRODUCTS\&m_id $=213 \&$ cntid $=90 \& A=S P$.

16. American Psychiatric Association . Diagnostic and statistical manual of mental disorders. 4th ed. Washington, DC: The Association; 2000.

17. Finley PR, Lee KC. Mood Disorders I: Major Depressive Disorders. In: Alldredge BK, Corelli RL, Ernst ME, editors. Koda-Kimble and Young's Applied Therapeutics: The clinical Use of Drugs. 10th ed. Philadelphia, PA: Lippincott Williams \& Wilkins; 2013. pp. 1949-82.

18. Bioequivalence and other unresolved issues in generic drug substitution. Meredith P. Clin Ther. 2003;25(11):2875

19. World Health Organization . Trade, foreign policy, diplomacy and health: Generic Drugs. WHO; 2011. Available from: http:// www.who.int/trade/glossary/story034/en/index.html.

20. US Food and Drug Administration Generic Drugs: Questions and Answers. Available from: http://www.fda.gov/drugs/resourcesforyou/consumers/questionsanswers/ucm100100.htm.

21. Generic versus branded pharmacotherapy in Parkinson's disease: does it matter? A review. Go CL, Rosales RL, Schmidt P, Lyons KE, Pahwa R, Okun MS. Parkinsonism Relat Disord. 2011;17(5):308

22. Therapeutic equivalency of generic antiepileptic drugs: results of a survey. Wilner AN. Epilepsy Behav. 2004;5(6):995.

23. Case reports of the reemergence of psychotic symptoms after conversion from brand-name clozapine to a generic formulation. Mofsen R, Balter J. Clin Ther. 2001;23(10):1720.

24. Factors associated with poor seizure control and increased side effects after switching to generic antiepileptic drugs. Bautista RE, Gonzales W, Jain D. Epilepsy Res. 2011;95(1-2):158.

25. Is generic fluoxetine effective? Yu BP, Chong YS, Maguire GA. J Affect Disord. 2004;81(2):185. 